\title{
Observing Lense-Thirring Precession in Tidal Disruption Flares
}

\section{Citation}

Stone, Nicholas, and Abraham Loeb. 2012. "Observing Lense-Thirring Precession in Tidal Disruption Flares." Physical Review Letters 108 (6). https://doi.org/10.1103/ physrevlett.108.061302.

\section{Permanent link}

http://nrs.harvard.edu/urn-3:HUL.InstRepos:41412143

\section{Terms of Use}

This article was downloaded from Harvard University's DASH repository, and is made available under the terms and conditions applicable to Open Access Policy Articles, as set forth at http:// nrs.harvard.edu/urn-3:HUL.InstRepos:dash.current.terms-of-use\#OAP

\section{Share Your Story}

The Harvard community has made this article openly available.

Please share how this access benefits you. Submit a story.

Accessibility 


\title{
Observing Lense-Thirring Precession in Tidal Disruption Flares
}

\author{
Nicholas Stone* and Abraham Loeb \\ Astronomy Department, Harvard University, 60 Garden St., Cambridge, MA 02138, USA
}

(Dated: October 29, 2018)

\begin{abstract}
When a star is tidally disrupted by a supermassive black hole (SMBH), the streams of liberated gas form an accretion disk after their return to pericenter. We demonstrate that Lense-Thirring precession in the spacetime around a rotating SMBH can produce significant time evolution of the disk angular momentum vector, due to both the periodic precession of the disk and the nonperiodic, differential precession of the bound debris streams. Jet precession and periodic modulation of disk luminosity are possible consequences. The persistence of the jetted X-ray emission in the Swift J164449.3+573451 flare suggests that the jet axis was aligned with the spin axis of the SMBH during this event.
\end{abstract}

PACS numbers: 98.62.Js, 98.62.Mw, 98.62.Nx

Introduction. The tidal disruption of a star by a supermassive black hole offers a unique opportunity to probe the nuclei of otherwise quiescent galaxies. However, the small number of candidate tidal disruption events (TDEs) makes it difficult to resolve theoretical uncertainties concerning their rates [1 $[\underline{6}]$, super-Eddington accretion phase [7 [9], and the period during which dissipation in shocks allows an accretion disk to form [10, 11].

An additional outstanding question about TDEs is whether or not they produce jets, as observed in many other accreting black hole systems. The past year has seen both the first theoretical models for TDE-associated jets [12, 13] and the discovery by the Swift satellite of an intense, transient gamma- and X-ray flare from a galactic nucleus at $z \approx 0.3514$. This flare has been explained by multiple authors [15 17] as jet emission from a TDE aligned with our line of sight (although alternate hypotheses exist [18]). A second possible TDE-associated jet was also recently observed [19].

If such jet emission is common, then TDEs provide a unique probe of the physics of accretion and jet production in the vicinity of distant black holes' horizons. Specifically, it is unknown at present whether jets will align with the black hole spin vector, the disk angular momentum vector, or some other component of the magnetic field geometry 20]. In most black hole accretion environments these directions are parallel, but the transient disk of a TDE will generally have some tilt with respect to the SMBH equatorial plane. In this Letter we demonstrate that if jets from tilted TDE accretion disks align with the disk normal vector, they will generally be expected to precess, often by observable amounts. Even absent the existence of a disk-aligned jet, or any jet at all, general relativistic (GR) effects will precess TDE disks with potentially observable consequences.

Spin evolution of a tilted disk. Stars of mass $M_{*}$ and radius $R_{*}$ that pass within a radius

$$
R_{\mathrm{t}}=R_{*}\left(M_{\mathrm{BH}} / M_{*}\right)^{1 / 3}
$$

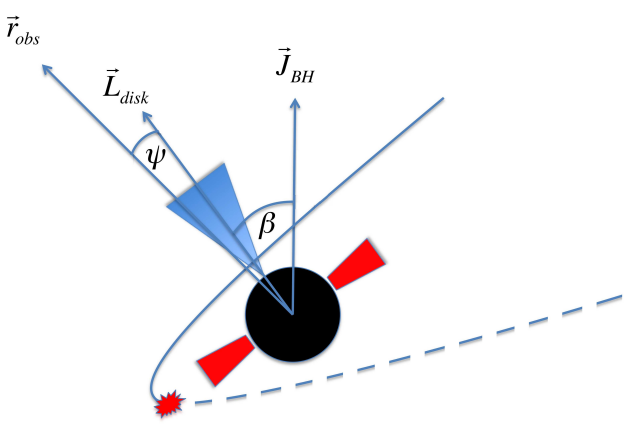

FIG. 1. Geometry of the tidal disruption of a star by a spinning SMBH. Following disruption of the star near its pericenter passage, an accretion disk will form in the star's orbital plane. As the disk precesses, the angle $\beta$ between the SMBH spin vector $\vec{J}_{\mathrm{BH}}$ and the disk angular momentum vector $\vec{L}_{\text {disk }}$ stays constant, but an associated jet may move relative to the observer's line of sight $\vec{r}_{\text {obs }}$.

of a black hole of mass $M_{\mathrm{BH}}$ will be tidally disrupted, with half their mass immediately unbound from the black hole [21]. For black holes of mass $M_{\mathrm{BH}} \gtrsim 10^{8} M_{\odot}$, the tidal radius $R_{\mathrm{t}}$ is inside the Schwarzschild radius $R_{\mathrm{S}}$ and stars are swallowed whole rather than disrupted. The bound debris rapidly expands and cools so that its pressure is negligible and the approximation of geodesic motion is accurate [10]. The most tightly bound debris stream of a star disrupted at radius $R_{\mathrm{P}}$ returns in a time

$$
t_{\text {fall }} \approx 50 M_{6}^{5 / 2} r_{\mathrm{p}}^{3} r_{*}^{-3 / 2} \mathrm{~s},
$$

where $M_{6}=M_{\mathrm{BH}} / 10^{6} M_{\odot}, r_{*}=R_{*} / R_{\odot}$ and $r_{\mathrm{p}}=R_{\mathrm{p}} / R_{\mathrm{S}}$ [8], although $t_{\text {fall }}$ depends on the stellar density profile and can be evaluated more precisely by numerical simulations 22. After a small multiple of this time, streamstream collisions circularize the returning gas and allow an accretion disk to form. In general, this transient accretion disk will not lie in the black hole equatorial plane.

An accretion disk inclined out of the equatorial plane of a spinning black hole by an angle $\beta$ (assumed to equal the inclination of the stellar orbit before disruption, $\beta_{*}$ 
- see Fig. 1) will be subject to Lense-Thirring torques with a strong radial dependence. For a thin disk 23], it is expected that the Bardeen-Petterson effect [24, 25] will induce a warp in the disk structure. However, for the thicker disks expected in many TDEs [8, 11], simulations combining GR and magnetohydrodynamic effects (GRMHD) have shown that the disk precesses as a solid body rotator [26, 27]. Such an accretion disk will precess with a period $T_{\text {prec }}=2 \pi \sin \beta(J / \tau)$, where $J$ is total angular momentum and $\tau$ is integrated torque. A notable feature of this formula is that $T_{\text {prec }}$ is independent of many disk model parameters, and depends only on the dimensionless radial surface density profile.

The simulations mentioned above considered disks with a roughly constant surface density. For surface densities of the form $\Sigma=\Sigma_{\mathrm{i}}\left(R / R_{\mathrm{i}}\right)^{-\zeta}$, the precession timescale is [26]

$T_{\text {prec }}=\frac{8 \pi G M_{\mathrm{BH}}(1+2 \zeta)}{c^{3}(5-2 \zeta)} \frac{r_{\mathrm{o}}^{5 / 2-\zeta} r_{\mathrm{i}}^{1 / 2+\zeta}\left(1-\left(r_{\mathrm{i}} / r_{\mathrm{o}}\right)^{5 / 2-\zeta}\right)}{a\left(1-\left(r_{\mathrm{i}} / r_{\mathrm{o}}\right)^{1 / 2+\zeta}\right)}$.

Here the disk inner $\left(R_{\mathrm{i}}\right)$ and outer $\left(R_{\mathrm{o}}\right)$ edges have been normalized to units of Schwarzschild radii $\left(r_{\mathrm{i}}=R_{\mathrm{i}} / R_{\mathrm{S}}\right.$, $\left.r_{\mathrm{o}}=R_{\mathrm{o}} / R_{\mathrm{S}}\right)$. The variable $a$ is the dimensionless black hole spin parameter, with values between 0 and 1 .

Whether or not the disks associated with tidal disruption flares approximately follow a surface density profile $\Sigma=\Sigma_{\mathrm{i}}\left(R / R_{\mathrm{i}}\right)^{-\zeta}$ is unclear. Ref. [8] presented a slim disk model for TDE accretion flows, with height $H$ given by:

$$
\frac{H}{R}=\frac{3 f}{4} \frac{10 \dot{M}}{\dot{M}_{\mathrm{Edd}}} \frac{R_{\mathrm{S}}}{R} K^{-1}
$$

where the function $K$ is defined as

$$
K=\frac{1}{2}+\sqrt{\frac{1}{4}+\frac{3 f}{2}\left(\frac{10 \dot{M}}{\dot{M}_{\mathrm{Edd}}}\right)^{2}\left(\frac{R_{\mathrm{S}}}{R}\right)^{2}} .
$$

Here $f=1-\left(R_{\mathrm{i}} / R\right)^{1 / 2} . \quad \dot{M} / \dot{M}_{\mathrm{Edd}}$ is the ratio of the mass accretion rate to the Eddington rate assuming $10 \%$ accretion efficiency.

However, this model is not suitable for use in calculating $T_{\text {prec }}$, as the zero-torque boundary condition used to calculate $f$ leads to an unphysical singularity in $\Sigma$ at $R_{\mathrm{i}}\left(\Sigma \propto R^{3 / 2} K^{2} / f\right)$. A different, numerical model was recently presented in Ref. [28], in which axisymmetric disk equations were evolved with a time-dependent rate of mass input at the pericenter of disruption. This model led to a shallow decline of $\Sigma$ with decreasing $r$ after the arrival of the inner edge of the accretion flow at the innermost stable circular orbit. Motivated by Refs. [8, 28], we consider $\zeta=-3 / 2,0,1$ in this paper. $T_{\text {prec }}$ increases by a factor $\approx 7$ when going from the $\zeta=1$ to the $\zeta=-3 / 2$ model.

The framework we followed is based on two underlying assumptions: (i) a coherent accretion flow exists;

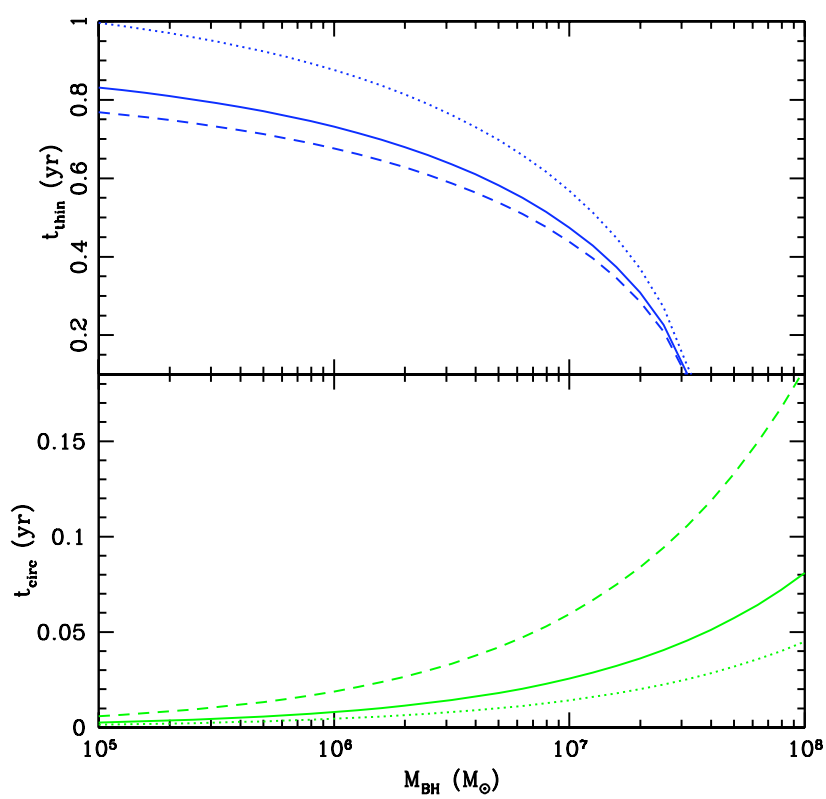

FIG. 2. Timescales for avoiding Bardeen-Peterson warping $t_{\text {thin }}$ (blue, top panel) and for establishing an accretion disk $t_{\text {circ }}$ (green, bottom panel) as functions of the black hole mass $M_{\mathrm{BH}}$. Dotted lines correspond to stars with a mass of $2 M_{\odot}$, solid lines to $1 M_{\odot}$ and dashed lines to $0.5 M_{\odot}$ (with a stellar mass-radius relationship adopted from Ref. [29], p. 208). We take $n_{\text {orb }}=3$ and $R_{\mathrm{p}}=0.5 R_{\mathrm{t}}$, and conservatively plot $t_{\text {thin }}$ for the outer edge of the disk, assuming $R_{\mathrm{o}}=2 R_{\mathrm{p}}$.

and (ii) the flow is not susceptible to Bardeen-Petterson warps $(H / R \gtrsim \alpha$, where $\alpha$ is the dimensionless disk viscosity parameter). Assumption (i) is only valid after a time $t_{\text {circ }} \approx n_{\text {orb }} t_{\text {fall }}$, where $n_{\text {orb }}$ is the number of orbits required to circularize the most tightly bound debris streams [11]. A value of $n_{\text {orb }} \sim 1-10$ is often assumed in the TDE literature, but this quantity is poorly constrained and could be higher for large $a$ and $\beta_{*}$, where Lense-Thirring precession can delay the stream-stream collisions necessary for disk formation [10]. At later times, assumption (ii) will break down, as $\dot{M} / \dot{M}_{\text {Edd }}$ declines and the disk becomes geometrically thinner.

Adopting Eq. (4) for convenience, $H / R$ will fall below $\alpha$ after a time

$$
\begin{array}{r}
t_{\text {thin }}=t_{\text {fall }}\left(\frac{5}{2} \frac{f}{X} \frac{M_{*} / t_{\text {fall }}}{\dot{M}_{\text {Edd }}} \frac{R_{\mathrm{S}}}{R}\right)^{3 / 5} \\
\approx 0.3 M_{6}^{2 / 5} r_{\mathrm{p}}^{6 / 5} m_{*}^{3 / 5} r_{*}^{-3 / 5}\left(\frac{f}{X_{-1}} \frac{R_{\mathrm{S}}}{R}\right)^{3 / 5} \mathrm{yr},
\end{array}
$$

where the function $X \sim \alpha$ and is $X=\alpha /\left(1-8 \alpha^{2} / 3 f\right)$. Also note that $X_{-1}=X / 0.1$. TDE disks will precess as solid body rotators during the time range $t_{\text {circ }}<t<t_{\text {thin }}$ as illustrated in Fig. 2, which shows that for $M_{\mathrm{BH}} \lesssim$ $10^{7} M_{\odot}$ (and any realistic $\left.R_{\mathrm{p}}\right)$, solid body precession will occur for $\lesssim 1$ yr. 
Angular momenta of returning debris streams. The evolution of the debris streams prior to their first return to pericenter has been studied in detail by Ref. [10]. The orbits of these streams, if non-equatorial, lack a constant orbital plane due to Lense-Thirring torques. The accretion disk is therefore fed by a supply of new gas with time-dependent angular momentum, which in turn evolves the direction of $\vec{L}_{\text {disk }}$. In contrast to direct precession of the accretion disk, we call this effect "differential stream precession," or DSP. Although we will compute numerical general relativistic solutions for the DSP, we can gain valuable intution from a simpler, lowest order estimate in the post-Newtonian limit.

The angle by which the angular momentum vector of a debris stream will precess during an orbit of period $T$ will be $\phi_{\text {orb }}(T) \approx \Delta \Omega \sin (\beta)=2 \pi \sin (\beta)\left(T / t_{\mathrm{LT}}\right)$, where $\Delta \Omega$ is the nodal precession and

$$
t_{\mathrm{LT}}=\frac{T}{2 a}\left(\frac{c^{2} A\left(1-e^{2}\right)}{G M_{\mathrm{BH}}}\right)^{3 / 2}
$$

is the Lense-Thirring precession period [30] for a gas stream of semimajor axis $A$ and eccentricity $e$. Defining $\Delta \phi_{\text {orb }}=\phi_{\text {orb }}(T)-\phi_{\text {orb }}(\infty)$ as a measure of the DSP,

$$
\Delta \phi_{\text {orb }}=4 \pi a \sin (\beta)\left(2 r_{\mathrm{p}}\right)^{3 / 2}\left((1+e)^{-3 / 2}-2^{-3 / 2}\right),
$$

which Taylor expands in the late-time, $R_{\mathrm{p}} / A \ll 1$ limit to $\Delta \phi_{\text {orb }} \approx 1.7 \sin (\beta) a r_{\mathrm{p}}^{-5 / 2} r_{*} M_{6}^{-1}\left(t / t_{\text {fall }}\right)^{-2 / 3}$.

Although Eq. (8) is not exact, it provides a valuable insight: the DSP is largest for low-mass, rapidly spinning SMBHs that disrupt stars with deeply plunging, inclined initial orbits. At early times the disk viscous timescale $t_{\text {visc }} \lesssim t_{\text {fall }}$ [8] so Eq. (8) approximates the angular evolution of $\vec{L}_{\text {disk }}$. We do not expect $\Delta \phi_{\text {orb }}>1^{\circ}$ after the establishment of a steady accretion flow $\left(t>3 t_{\text {fall }}\right)$ for any TDEs with solar-type stars and $M_{6} \gtrsim 2$, although these constraints relax for stars with $r_{*}>1$.

To obtain an exact solution for the time evolution of angular momentum in the returning debris streams, a GR calculation is needed. We numerically integrate the Kerr geodesic equations following the formalism of Ref. 31]. We assume a flat distribution of debris mass with specific Newtonian energy $E$, a spread in that energy of $3 G M_{\mathrm{BH}} R_{*} / R_{\mathrm{p}}^{2}$ [8], and obtain constants of integration for each debris stream by transforming the initial conditions $\left\{E, R_{\mathrm{p}}, \beta\right\}$ to $\left\{E_{\mathrm{GR}}, L_{\mathrm{z}}, Q\right\}\left(E_{\mathrm{GR}}, L_{\mathrm{z}}, Q\right.$ are specific energy, z-component angular momentum, and Carter's constant for Kerr metric test particles). Good agreement with Eq. (8) is shown in Fig. 3 .

Observational implications. We have shown that the Lense-Thirring effect will cause the direction of a TDE disk's angular momentum vector to evolve in time. Direct precession of the accretion disk is the dominant effect, but in some cases DSP can cause a significant nonperiodic evolution in $\vec{L}_{\text {disk }}$. The precession of the ac-

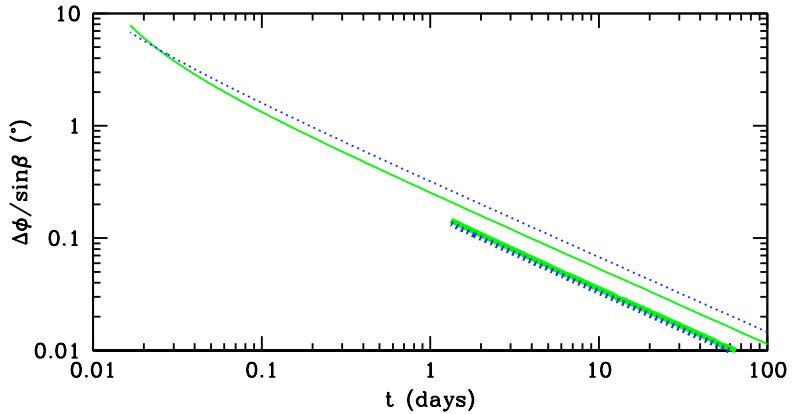

FIG. 3. The angular shift $\Delta \phi_{\text {orb }}$. The thick curves illustrate the disruption of a solar-type star with $M_{\mathrm{BH}}=10^{6} M_{\odot}, a=$ 0.8 , and $r_{\mathrm{p}}=13$; the thin curves are the same but with $r_{\mathrm{p}}=3$. The blue dotted lines are Eq. (8), while the green solid lines are numerical geodesic solutions. The curves do not extend prior to $t=t_{\text {fall }}$, and are normalized by $\sin \beta$.

cretion disk will modulate the observed disk luminosity at least by a factor of $\cos (\psi)$, and lead to periodic pulsations of the associated transient quasar. This periodic modulation could in principle be extracted from the Fourier decomposition of a TDE lightcurve, but perhaps a more promising avenue for detection lies in the fraction of events for which the disks will precess into an edge-on phase. This could reduce the observed disk flux by $\sim 2$ orders of magnitude while simultaneously reddening the peak emission frequency [11]. Even in the absence of jet emission, observations of a "blinking" TDE flare could provide strong evidence of precession and allow both $a$ and the disruption parameters to be constrained.

The most exciting possible consequence, however, is precession of jets associated with TDE disks. If we assume that relativistic jets in tilted accretion systems align with $\vec{L}_{\text {disk }}$, narrow jets will precess out of the observer's line of sight in a small fraction of $T_{\text {prec }}$. Continuous observation of a jet for a relatively short period of time, $t_{\mathrm{obs}}$, allows very strong constraints to be placed on combinations of $a$ and disruption parameters such as $r_{\mathrm{p}}$ and $\beta_{*}$ (assuming still that $\beta=\beta_{*}$ ). Alternatively, repeated observation of TDE-associated jets could serve as evidence that jets align with $\vec{J}_{\mathrm{BH}}$ or an aspect of the magnetic field geometry, provided that sufficient non-precession is observed. We note that the DSP, though generally subdominant, can in some cases cause very rapid precession (up to $\sim 0.1^{\circ} / \mathrm{min}$ ) at the onset of the flare (Fig. 3). If an associated jet is aligned with $\vec{L}_{\text {disk }}$, this will lead to a brief, nonrepeating transient which could fake an unusually long gamma ray burst provided $\theta_{\text {jet }} \lesssim 1^{\circ}$.

To provide a concrete example of the above considerations, we consider the tidal disruption candidate Swift J164449.3+573451, for which Ref. 15] inferred the following relevant disruption parameters: $M_{\mathrm{BH}} \sim 10^{5}-$ $10^{6} M_{\odot}, R_{\mathrm{p}} \approx 13 R_{\mathrm{S}} M_{6}^{-5 / 6}$, and $\theta_{\text {jet }} \sim 10^{-1.5}\left(\theta_{\text {jet }}\right.$ is estimated from both comparing the theoretical TDE rate 


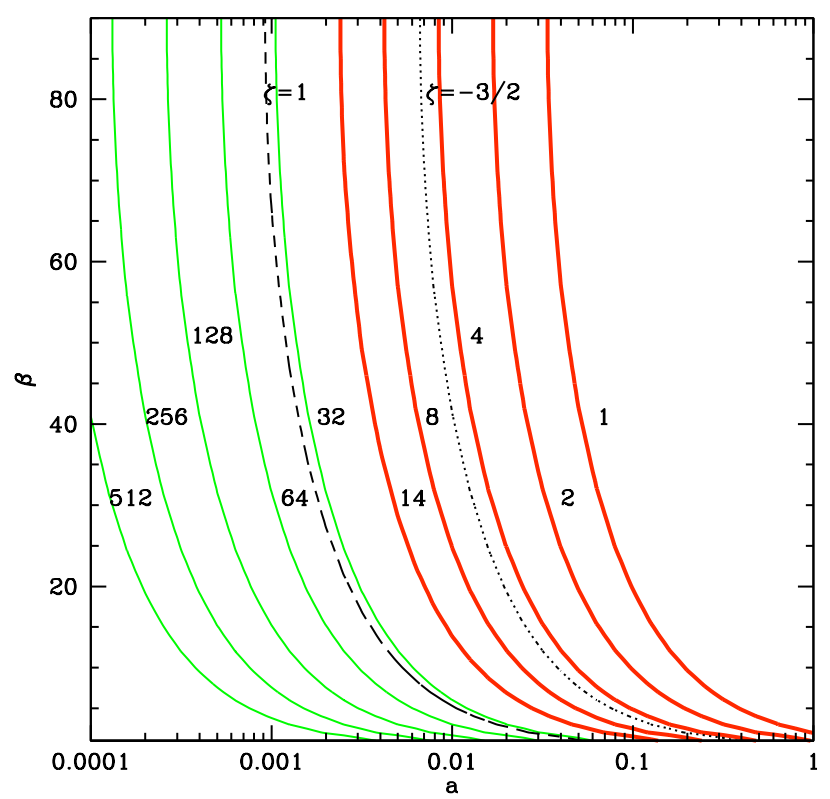

FIG. 4. Regions of $a-\beta$ parameter space that can be excluded by continuous observations of a TDE jet with the inferred parameters in Ref. [15] and $\zeta=0$. The solid curves show contours of constant $t_{\text {obs }}=T_{\text {prec }} \times 2\left(\theta_{\text {jet }} / 10^{-1.5}\right) /(2 \pi \sin \beta)$ : the maximum number of days it would take for a jet initially in the observers' line of sight to precess off-axis, with the jet opening angle normalized to $10^{-1.5}$. We take $R_{\mathrm{o}}=2 R_{\mathrm{p}}$ and $R_{\mathrm{i}}=3 R_{\mathrm{S}}$. Regions of parameter space to the right of the thick red contours can be excluded for the Swift TDE jet, which exhibited bright X-ray emission for over two weeks. The 14 day contours for $\zeta=-3 / 2$ and $\zeta=1$ are shown with black dotted and dashed lines, respectively. The effect of the DSP is negligible for these parameters, and neglected here.

to the observed rate of jets over the period of the Swift mission, and the Eddington limit of the $\mathrm{SMBH}$ ).

Figure 4 shows the resulting constraints on the joint $a$ $\beta$ parameter space of this TDE if we take $M_{6}=1$. Since the bright X-ray emission from Swift J164449.3+573451 persisted for over two weeks, at least one of the following statements must be true: (i) the value of $a$ is extremely low, $\lesssim 10^{-2}\left(10^{-1}\right.$ if $\left.\zeta=-3 / 2\right)$; (ii) the initial orbit of the disrupted star was tightly aligned to within $\sim \theta_{\text {jet }}$ of the black hole equatorial plane; or (iii) the jet emission was not aligned with the disk spin axis. The first possibility would represent an unusually low value of black hole spin and could be excluded if the BlandfordZnajek mechanism was responsible for jet launching [32], while (ii) requires that there will be a larger abundance of somewhat shorter events. Since such flares are not frequently observed, the persistent X-ray emission in Swift J164449.3+573451 suggests that its jet was aligned with the steady spin axis of the black hole rather than with its precessing disk. Future GRMHD simulations can test this inference from first principles. The detection of ad- ditional TDE-associated jets in future surveys would test the statistical robustness of this conclusion.

Acknowledgments. This work was supported in part by NSF grant AST-0907890 and NASA grants NNX08AL43G and NNA09DB30A. We thank Ashley Zauderer and both anonymous referees for useful comments.

* nstone@cfa.harvard.edu

[1] Donley, J. L., Brandt, W. N., Eracleous, M., \& Boller, T. 2002, AJ, 124, 1308

[2] Wang, J., \& Merritt, D. 2004, ApJ, 600, 149

[3] Merritt, D., \& Poon, M. Y. 2004, ApJ, 606, 788

[4] Perets, H. B., Hopman, C., \& Alexander, T. 2007, ApJ, 656,709

[5] Gezari, S., Basa, S., Martin, D. C., et al. 2008, ApJ, 676, 944

[6] van Velzen, S., Farrar, G. R., Gezari, S., et al. 2010, arXiv:1009.1627

[7] Loeb, A., \& Ulmer, A. 1997, ApJ, 489, 573

[8] Strubbe, L. E., \& Quataert, E. 2009, MNRAS, 400, 2070

[9] Strubbe, L. E., \& Quataert, E. 2011, MNRAS, 415, 168

[10] Kochanek, C. S. 1994, ApJ, 422, 508

[11] Ulmer, A. 1999, ApJ, 514, 180

[12] Giannios, D., \& Metzger, B. D. 2011, MNRAS, 1137

[13] van Velzen, S., Körding, E., \& Falcke, H. 2011, MNRAS, L310

[14] Levan, A. J., Tanvir, N. R., Cenko, S. B., et al. 2011, Science, 333, 199

[15] Zauderer, B. A., Berger, E., Soderberg, A. M., et al. 2011, Nature, 476, 425

[16] Bloom, J. S., Giannios, D., Metzger, B. D., et al. 2011, Science, 333, 203

[17] Burrows, D. N., Kennea, J. A., Ghisellini, G., et al. 2011, Nature, 476, 421

[18] Quataert, E., \& Kasen, D. 2011, arXiv:1105.3209

[19] Cenko, S. B., Krimm, H. A., Horesh, A., et al. 2011, arXiv:1107.5307

[20] Fragile, P. C. 2008, Microquasars and Beyond

[21] Rees, M. J. 1988, Nature, 333, 523

[22] Laguna, P., Miller, W. A., Zurek, W. H., \& Davies, M. B. 1993, ApJL, 410, L83

[23] Kumar, S., \& Pringle, J. E. 1985, MNRAS, 213, 435

[24] Bardeen, J. M., \& Petterson, J. A. 1975, ApJL, 195, L65

[25] Papaloizou, J. C. B., \& Pringle, J. E. 1983, MNRAS, 202, 1181

[26] Fragile, P. C., Blaes, O. M., Anninos, P., \& Salmonson, J. D. 2007, ApJ, 668, 417

[27] Dexter, J., \& Fragile, P. C. 2011, ApJ, 730, 36

[28] Montesinos Armijo, M., \& de Freitas Pacheco, J. A. 2011, ApJ, 736, 126

[29] Kippenhahn, R., \& Weigert, A. 1994, Stellar Structure and Evolution, XVI, 468 pp. 192 figs. Springer-Verlag Berlin Heidelberg New York. Also Astronomy and Astrophysics Library

[30] Merritt, D., Alexander, T., Mikkola, S., \& Will, C. M. 2010, Phys. Rev. D, 81, 062002

[31] Drasco, S., \& Hughes, S. A. 2004, PRD, 69, 044015

[32] Lei, W.-H., \& Zhang, B. 2011, ApJL, 740, L27 